\title{
Nematic Ordering Pattern Formation in the Process of Self-Organization of Microtubules in a Gravitational Field
}

\author{
Hu Jian • Qiu Xijun • Li Ruxin
}

Published online: 9 September 2007

(C) Springer Science + Business Media B.V. 2007

\section{Erratum to: J Biol Phys (2006) 32: 497-506 DOI 10.1007/s10867-006-9032-x}

The authors of this paper wish to retract the article as they have carelessly (although unintentionally) copied parts of the following papers:

S. Portet, J.A. Tuszynski, J.M. Dixon and M.V. Sataric, Models of spatial and orientational self-organization of microtubules under the influence of gravitational fields, Physical Review E 68, 021903-1-9 (2003)

J.A. Tuszynski, M.V. Sataric, S. Portet and J.M. Dixon, Gravitational symmetry breaking leads to a polar liquid crystal phase of microtubules in vitro, Journal of Biological Physics 31(3-4), pp. 477-486 (2005)

V.A. Baulin, Self-assembled aggregates in the gravitational field: growth and nematic order, Journal of Chemical Physics 119(5), pp. 2874-2885 (2003)

V.A. Baulin and A. R. Khokhlov, Nematic ordering of rigid rods in a gravitational field, Physical Review E 60(3), pp. 2973-2977 (1999)

J.A. Tuszynski, M.V. Sataric, S. Portet and J.M. Dixon, Physical interpretation of microtubule self-organization in gravitational fields, Physics Letters A 340, pp. 175-180 (2005)

The authors deeply regret the publication of the article and are hereby withdrawing it.

The online version of the original article can be found at http://dx.doi.org/10.1007/s10867-006-9032-x

H. Jian $(\square) \cdot$ Q. Xijun

Department of Physics, Shanghai University, Shanghai 200444, China

e-mail: shhujian2005@yahoo.com.cn

L. Ruxin

Shanghai Institute of Optics and Fine Mechanics, Chinese Academy of Science,

Shanghai 201800, China 